OPEN ACCESS

Edited by:

Giulia Morra,

Istituto di Scienze e Tecnologie Chimiche Giulio Natta (SCITEC), Italy

Reviewed by:

Giovanni La Penna,

National Research Council (CNR), Italy

Giorgio Colombo,

University of Pavia, Italy

*Correspondence:

Martin Zacharias

martin.zacharias@mytum.de

Specialty section:

This article was submitted to

Theoretical and Computational

Chemistry,

a section of the journal

Frontiers in Chemistry

Received: 16 June 2020 Accepted: 08 September 2020

Published: 08 October 2020

Citation:

Santini BL and Zacharias M (2020) Rapid in silico Design of Potential Cyclic Peptide Binders Targeting

Protein-Protein Interfaces.

Front. Chem. 8:573259.

doi: 10.3389/fchem.2020.573259

\section{Rapid in silico Design of Potential Cyclic Peptide Binders Targeting Protein-Protein Interfaces}

\author{
Brianda L. Santini and Martin Zacharias* \\ Physics Department T38, Technical University of Munich, Garching, Germany
}

Rational design of specific inhibitors of protein-protein interactions is desirable for drug design to control cellular signal transduction but also for studying protein-protein interaction networks. We have developed a rapid computational approach to rationally design cyclic peptides that potentially bind at desired regions of the interface of protein-protein complexes. The methodology is based on comparing the protein backbone structure of short peptide segments (epitopes) at the protein-protein interface with a collection of cyclic peptide backbone structures. A cyclic peptide that matches the backbone structure of the segment is used as a template for a binder by adapting the amino acid side chains to the side chains found in the target complex. For a small library of cyclic peptides with known high resolution structures we found for the majority ( $\sim 82 \%)$ of 154 protein-protein complexes at least one very well fitting match for a cyclic peptide template to a protein-protein interface segment. The majority of the constructed protein-cyclic peptide complexes was very stable during Molecular Dynamics simulations and showed an interaction energy score that was typically more favorable compared to interaction scores of typical peptide-protein complexes. Our cPEPmatch approach could be a promising approach for rapid suggestion of cyclic peptide binders that could be tested experimentally and further improved by chemical modification.

Keywords: protein-protein complexes, protein interaction inhibition, protein binding modulation, cyclo peptide design, drug design with cyclo-peptides, rational cyclo peptide binders

\section{INTRODUCTION}

Protein-protein interactions (PPIs) serve as the basis for nearly all biological processes, they play key roles in intercellular communication, cell-to-cell signaling, metabolic and developmental control, and programmed cell death (Fontaine et al., 2015). Abnormal regulation of PPIs results in many human diseases such as cancers, immune disorders, and neurodegenerative diseases. Modulating these aberrant interactions is of clinical relevance, however, targeting PPIs may be challenging because of the intrinsic properties of protein-protein interfaces (Ryan and Matthews, 2005; Villoutreix et al., 2014).

PPI interfaces typically involve large, flat binding sites which are devoid of any major binding pocket (Conte et al., 1999; Bahadur and Zacharias, 2008), making them challenging targets for conventional drug modalities because small molecules generally do not bind to large binding sites with high affinity. Larger molecules with more extended binding surfaces, e.g., monoclonal antibodies, cannot easily cross the cell membrane to reach the targets (Qian et al., 2017). Progress has, however, been made in targeting protein-protein interactions (PPI) by realizing that not all 
the surface area of a PPI contributes equally to the strength of the interaction between the protein partners (Keskin et al., 2005; Wells and McClendon, 2007; Arkin et al., 2014). Their binding is mediated by strong packing or electrostatic interactions where only a few neighboring amino acids are crucial for binding and recognition. These small areas of disproportionately highaffinity binding at the protein-protein interface are referred to as "hot spots" (Keskin et al., 2005; Metz et al., 2012). Nevertheless, targeting specifically protein-protein interaction hot spots still tends to require complex drug molecules (Fry, 2006).

A promising approach for PPI targeting is the design of molecules that closely mimic epitopes, i.e., binding segments, of a partner protein found as part of the interface. These molecules are typically derived from existing peptides and tend to conserve a protein-like chain, but with its chemical structure modified to adjust the molecular properties to become more drug-like. Following this strategy, many investigators have turned their attention to macrocycles, particularly cyclic peptides, as potential PPI inhibitors (Driggers et al., 2008; Marsault and Peterson, 2011; Mallinson and Collins, 2012). Cyclic peptides are among the most promising PPI modulators owing to their ability to potentially bind to large surfaces with reasonable affinity and specificity and due to the enhanced stability and bioavailability as compared to linear peptides (Grauer and König, 2009; Gavenonis et al., 2014; Nevola and Giralt, 2015). The advantages of cyclic peptides in terms of conformational rigidity and proteolytic resistance can also be further enhanced by modifying the peptide backbone. Proteases act upon cleavage sites in the peptide sequence, which may be at the $\mathrm{N}$ or $\mathrm{C}$-terminus, or sometimes at a particular motif within the peptide. Peptide cyclization often reduces the accessibility to cleavage sites in proteases. There is also evidence that cyclic peptides may have improved membrane permeability, compared with linear peptides, in cases where the cyclic peptide can internally satisfy its hydrogen bonds (Rezai et al., 2006).

Early and recent work has demonstrated the possibility to use cyclic peptides that could bind at protein-protein interface regions and interfere with protein-protein binding (Schreiber and Crabtree, 1992; Dechantsreiter et al., 1999; Sulyok et al., 2001; Shi et al., 2004; Zhang et al., 2014; Siegert et al., 2016; Shin et al., 2017). Identification of potential appropriate cyclic peptides can be achieved using techniques used in small-molecule drug design efforts such as virtual screening and pharmacophore matching approaches (Zhang et al., 2014; Duffy et al., 2015; Qian et al., 2017). In this study, we propose a straight forward automated process for the rapid search and optimization of cyclic peptides as protein-protein interaction inhibitors based on known structures of cyclic peptides. Our main goal is to start with a library of existing cyclic peptides and use them as templates for the construction of molecules that closely mimic the epitopes of a partner protein in PPI complexes. We start our process by characterizing backbones motifs of the backbone of epitopes found on the PPI interfaces and comparing them to backbone motifs in a data base of cyclic peptides. If a backbone match is found, the cyclic peptide structure is superimposed and the corresponding interface amino acids of the cyclic peptides are replaced by those of the binding epitope, to closely mimic the specific interface. The generated cyclo-peptide-protein complexes are refined and evaluated by Molecular Dynamics (MD) simulations and energetic evaluation. In this proof-ofprinciple study our automated approach is tested on 154 proteinprotein complexes. For the majority of $\sim 71 \%$ we identify cyclic peptide motifs that result in stable complexes during $\mathrm{MD}$ refinement and closely mimic the natural interface epitope.

\section{MATERIALS AND METHODS \\ Cyclic Peptide Matching (cPEPmatch) Approach}

Many protein-protein interactions are at least in part mediated by short linear motifs that can be part of $\alpha$-helices, $\beta$-strands or loops (London et al., 2010). The workflow of the cPEPmatch that we propose in this work is based on the idea that the shape of the backbone structure of the PPI interfaces plays a key role in the binding interaction between these short motifs (Kallen et al., 1998). Our cyclic peptide matching approach is divided into 4 main steps (outlined in Figure 1) including a final refinement and evaluation step using the AmberTools 18 package (Case et al., 2005). The tool backbo characterizes the backbones of each cyclic peptide in terms of distances between backbone atoms of 4 consecutive residues, creating the motifs that are then stored in a data base. A second tool, int_analysis, identifies all the neighboring protein residues from the PPI complexes as the interface and, subsequently, characterizes its backbone atom motifs also in sets of four consecutive residues and identifies matching backbone motifs in the cyclic peptide data file. For each protein-protein interface motifs within up to $7 \AA$ distance from the partner protein were considered. For the matching of backbone atom distances of a motif at the protein-protein interface and a corresponding motif in a cyclic peptide an average distance deviation of up to $0.5 \AA$ was used as threshold to accept only sufficiently precise matches (in the following termed F-RMSD). In a third step a cyclic peptide is superimposed on the matching backbone segment of a partner protein to form an appropriate binding placement. This step returns a coordinate file of the cyclic peptide in complex with the receptor partner protein. The side chains of the four consecutive (matching) residues at the interface between cyclic peptide and protein are then replaced by the side chains found in the original protein-receptor complex. Exceptions for residue substitutions are made when the matched residue of the cyclic peptide is one involved in a disulfide bond that is part of the peptide cyclization (not changed). The resulting complex was further refined and evaluated by energy minimization and Molecular Dynamics (MD) simulation (see below).

\section{Cyclic Peptide Library and Protein-Protein Complexes}

Different chemical strategies can be used to create cyclized peptides, including head-to-tail, disulfide, other side-chain to side-chain, and side-chain to terminus bonding (Martins and Carvalho, 2007; Wells and McClendon, 2007; Huigens et al., 2013; Duffy et al., 2015; Kuenemann et al., 2015; Milhas et al., 2016). Incorporating multiple cyclizations to generate peptides 


\section{Cyclic Peptide Database Motif Construction}

Each Structural motif is characterized by the distances between 4 consecutive $\mathrm{Ca}$ atoms in the cyclic peptide.

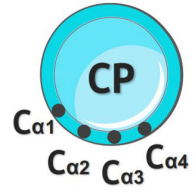

\section{PPI Interface Characterization}

Determines the distances of 4 consecutive $C \alpha$ atoms at the interface and finds all matching motifs in the Cyclic Peptide database.

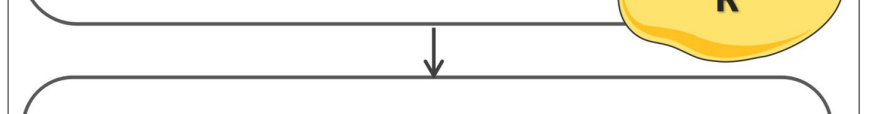

\section{Match Superimpose}

Superimposes the motifs of the matched Cyclic Peptides to the motif of the Ligand. Returns a Fit-RMSD value.

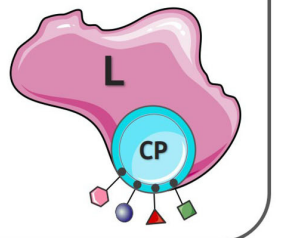

\section{Sequence Adaptation}

The sequence of each matched Cyclic Peptide at the interface is changed to the corresponding amino acids of the $(L)$ partner, followed by Energy Minimization and Molecular Dynamics refinement.

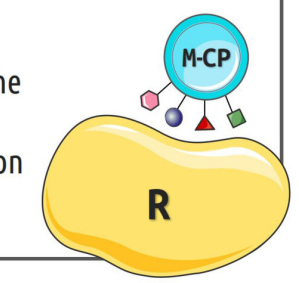

FIGURE 1 | Workflow of the in silico cyclic peptide binder construction. CP indicates cyclic peptide, $L$ and $R$ represent the two partners of $a$ protein-protein interaction pair.

that are bicyclic, tricyclic, etc., can provide additional restraints to rigidify the peptide and provide further complexity of design space (Villoutreix et al., 2014; Che, 2019; Duffy et al., 2019). We extracted structures of 30 cyclic peptides that vary in cyclization type and sizes as representative set of cyclic peptides with known well resolved 3D structure. The cyclic peptides structure types have been chosen to represent common portions of protein-protein interaction hot-spots such as $\alpha$-helices, $\beta$-strands, and turns. All structures used were downloaded from the Protein Data Bank (PDB) and a complete list is found in Supplementary Table 1. We applied the method to the analysis of protein-protein complexes chosen from the protein-protein interface (PPI) Benchmark constructed by Tikk and collaborators
(Tikk et al., 2010), the complete list of complexes is given in Supplementary Table 2.

\section{Molecular Dynamics Simulation and MM/GBSA Evaluation}

All cyclic peptide-protein complexes were processed for energy minimization and MD simulations using the tleap module of Amber18. Protein parameters were retrieved from the ff14SB force field (Maier et al., 2015). The complexes were neutralized by the addition of $\mathrm{Na}+$ or $\mathrm{Cl}$ - ions and solvated in an orthorhombic box with a minimum distance to box-boundaries of $10 \AA$ using explicit TIP3P water molecules (Jorgensen et al., 1983). All simulation systems were first energy minimized with the steepest descent method in 2000 steps by using the Amber18 Sander module. All subsequent MD simulations were performed with the pmemd.cuda module. Initially, the systems were heated up to $310 \mathrm{~K}$ in three stages (in $100 \mathrm{~K}$ steps). Each stage was simulated for $100 \mathrm{ps}$ and included positional restraints on all non-hydrogen atoms with respect to the starting conformation. Subsequently, positional restraints were gradually reduced from 25 to 0.5 $\mathrm{kcal} \cdot \mathrm{mol}^{-1} \cdot \mathrm{A}^{-2}$ in five consecutive simulations of $100 \mathrm{ps}$ at 310 $\mathrm{K}$ and at constant pressure of 1 bar. The equilibrated structures served as input for the production runs for each system, with no restraints. Data gathering simulations were carried out for $5 \mathrm{~ns}$. Coordinates were written out every 500 steps. A time step of 2 fs was used and all bonds involving hydrogens were constrained to the optimal length using Shake (Ryckaert et al., 1977). In order to estimate the stability of a bound cyclic peptide the L-RMSD (root-mean-square deviation of all non-hydrogen atoms of the cyclic peptide after best superposition of the receptor binding site on the start structure) was calculated to assess the deviation of peptide ligand along the $5 \mathrm{~ns}$ of trajectory for each match. For the superposition on the receptor structure the receptor binding site that is all atoms within $6 \AA$ of any peptide ligand atom was considered.

MM/GBSA (molecular mechanics Generalized Born surface area) binding free energy calculations were carried out to estimate the strength of cyclopeptide-receptor interactions (Chen et al., 2016; Wang et al., 2018). Binding free energies were estimated using 250 snapshots retrieved from the last $2.5 \mathrm{~ns}$ of MD simulation production using the MMPBSA.py module in Amber18. GB calculations were carried out using the modified GB model (igb=5) with mbondi2, and $\alpha, \beta$, and $\gamma$ values of 1.0 , 0.8 , and 4.85 , respectively. Dielectric constants for the solvent and the solute were set to 80 and 5 , respectively.

\section{RESULTS AND DISCUSSIONS}

Identification of cyclic peptides that bind to the interface region of protein-protein complexes may provide a promising route for modulating PPIs (Qian et al., 2017). In this work, we present an automated rapid workflow (cPEPmatch) that identifies and optimizes cyclic peptides as starting templates to construct modulators of protein-protein interactions. It is, based on the matching of small backbone motifs at protein-protein interfaces and corresponding motifs in cyclic peptides (Figure 1). It has 


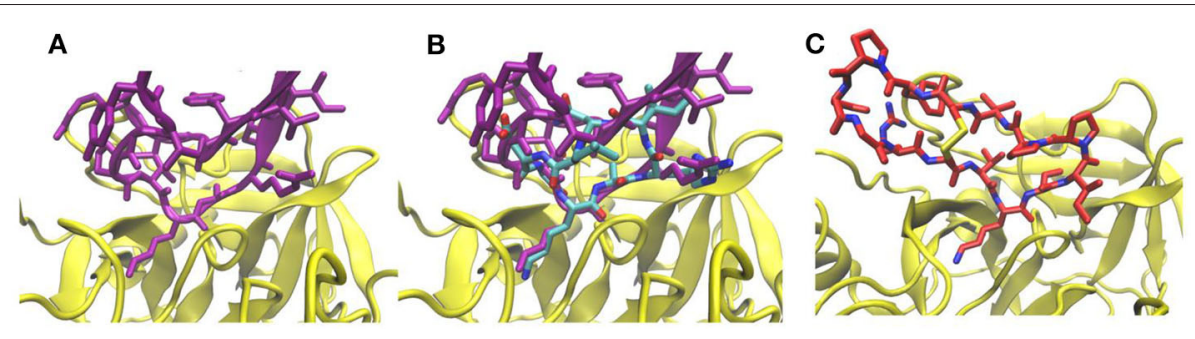

FIGURE 2 | (A) Interface structure of the complex (pdb4dg4) of trypsin inhibitor protein (pink) and trypsin (yellow). (B) Same view as in (A) but with a superimposed cyclic peptide (pdb3avb, atom color coded) and adapted interface side chains after 5 ns MD simulation. (C) Complex of sunflower cyclic peptide (red/blue stick model) and trypsin (pdb1sfi).

been observed that many protein-protein interaction surfaces are dominated by short segments of peptides (London et al., 2010) and that the constrained backbone often plays a key role in the binding interactions (Kallen et al., 1998).

We first tested the approach on a complex formed by bovine trypsin inhibitor (BPTI) protein and the trypsin protease (pdb4dg4). Our matching approach identified a cyclic peptide backbone that closely resembled the protein backbone of the BPTI protein at the interface (Figures 2 A,B) with a root-meansquare deviation (RMSD) of the 4 residue backbone segment of only $0.4 \AA$ with respect to the corresponding segment in the BPTI protein. The side chain replacement resulted in excellent sterical fit closely resembling the original interface structure even after the MD simulation refinement in explicit solvent. The predicted structure of the cyclic-peptide trypsin complex is also in excellent agreement with an experimentally known structure of the sunflower cyclic peptide in complex with trypsin (pdb1sfi, Figure 2C, RMSD of the interface segment $<0.5 \AA$, Table $\mathbf{1}$ ). Note, that the sunflower peptide is not included in our cyclic peptide data base. The evaluation of the cyclic peptide interaction with trypsin resulted in a MMGBSA interaction energy of $43.8 \mathrm{kcal} \cdot \mathrm{mol}^{-1}$ (Table 1). This is less than what is obtained for the sunflower peptide $\left(-68.8 \mathrm{kcal} \cdot \mathrm{mol}^{-1}\right)$ but the sunflower peptide is also significantly larger and forming a more extended interface compared to the generated potential small cyclic peptide binder. The calculated MMGBSA interaction energy for this test case was significantly more favorable than the MMGBSA interaction energy obtained for a typical peptide-protein complex with binding affinities in the micromolar regime (Table 1).

Next we applied our cPEPmatch approach systematically to 154 protein-protein complexes with known structures to identify putative matches that could potentially bind at the PP interface and interfere with PP interaction. According to our threshold criteria (see Methods) we were able to identify at least one putative cyclic peptide match for $82 \%$ of all the cases studied (Table 1). The putative cyclic peptides matched with the best RMSD and sterical fit to each PPI were superimposed, interface residues were substituted and the interface structure was refined by short MD simulations in explicit solvent. We found that during the $5 \mathrm{~ns}$ of $\mathrm{MD}, 86 \%$ of the matched complexes were stable (no dissociation and L-RMSD $<5 \AA$ ). Hence, the overall yield of stable predicted complexes of cyclic peptides with protein binding partners was $\sim 71 \%\left(0.82^{\star} 0.86\right)$. These
TABLE 1 | Performance of the cyclo-peptide matching approach.

\section{MM/GBSA Comparison}

Trypsin-Sunflower Cyclic Peptide Inhibitor (1sfi)

$-68.85 \mathrm{kcal} \cdot \mathrm{mol}^{-1}$

Trypsin-Matched Cyclic Peptide (3avb)

FBP11 WW Domain (1ywi)

$-43.79 \mathrm{kcal} \cdot \mathrm{mol}^{-1}$

FE65 WW Domain (2ho2)

$-30.06 \mathrm{kcal} \cdot \mathrm{mol}^{-1}$

$-32.02 \mathrm{kcal} \cdot \mathrm{mol}^{-1}$

\section{Overall Performance}

Putative match found

$82 \%$

Stable binding during MD

$86 \%$

Average F-RMSD of best matches

Average L-RMSD of best matches

$0.38 \AA$

$2.77 \AA$

Average MM/GBSA of best matches

$-43.09 \mathrm{kcal} \cdot \mathrm{mol}^{-1}$

identified complex structures had an average F-RMSD, L-RMSD and MMGBSA interaction energy of $0.38 \AA$, $2.77 \AA$, and -43.09 $\mathrm{kcal} \mathrm{mol}^{-1}$, respectively.

Examples of predicted complexes between cyclic peptides that mimic a $\beta$-hairpin, a turn and an $\alpha$-helical interface segment and partner proteins pdb1cgI, pdb2hle, and pdb2nz8, respectively, are illustrated in Figures 3A-C. A more detailed list of the top 30 matches in terms of calculated MMGBSA interaction energy is found in Table 2. The L-RMSD of all these complexes is in between 1 and $5 \AA$. The calculated MM/GBSA interaction energies vary significantly from system to system (from $\sim-80$ to $-30 \mathrm{kcal} \mathrm{mol}^{-1}$ ) which might be due to different sizes but may also be due to the targeted interface region in each complex. However, the MMGBSA interaction energies are in most cases more favorable compared to small linear peptides that binding with micromolar binding constants to target domains (Table 1).

To assess the consistency of our simulations and MMGBSA evaluation, we expanded our original MD productions by 5 ns and also performed a second independent set of MD simulations (of $10 \mathrm{ns)}$ ) for each of the highlighted systems in our study, 1cgi, $2 \mathrm{hle}, 2 \mathrm{nz} 8$, and $4 \mathrm{dg} 4$ (that represent typical $\beta$-hairpin, a turn and an $\alpha$-helical interfaces). For each case and each simulation MMGBSA binding free energy calculations were calculated using 250 snapshots retrieved from the three time intervals corresponding to $2.5-5.0,5.0-7.0$, and $7.5-10$ ns of every 

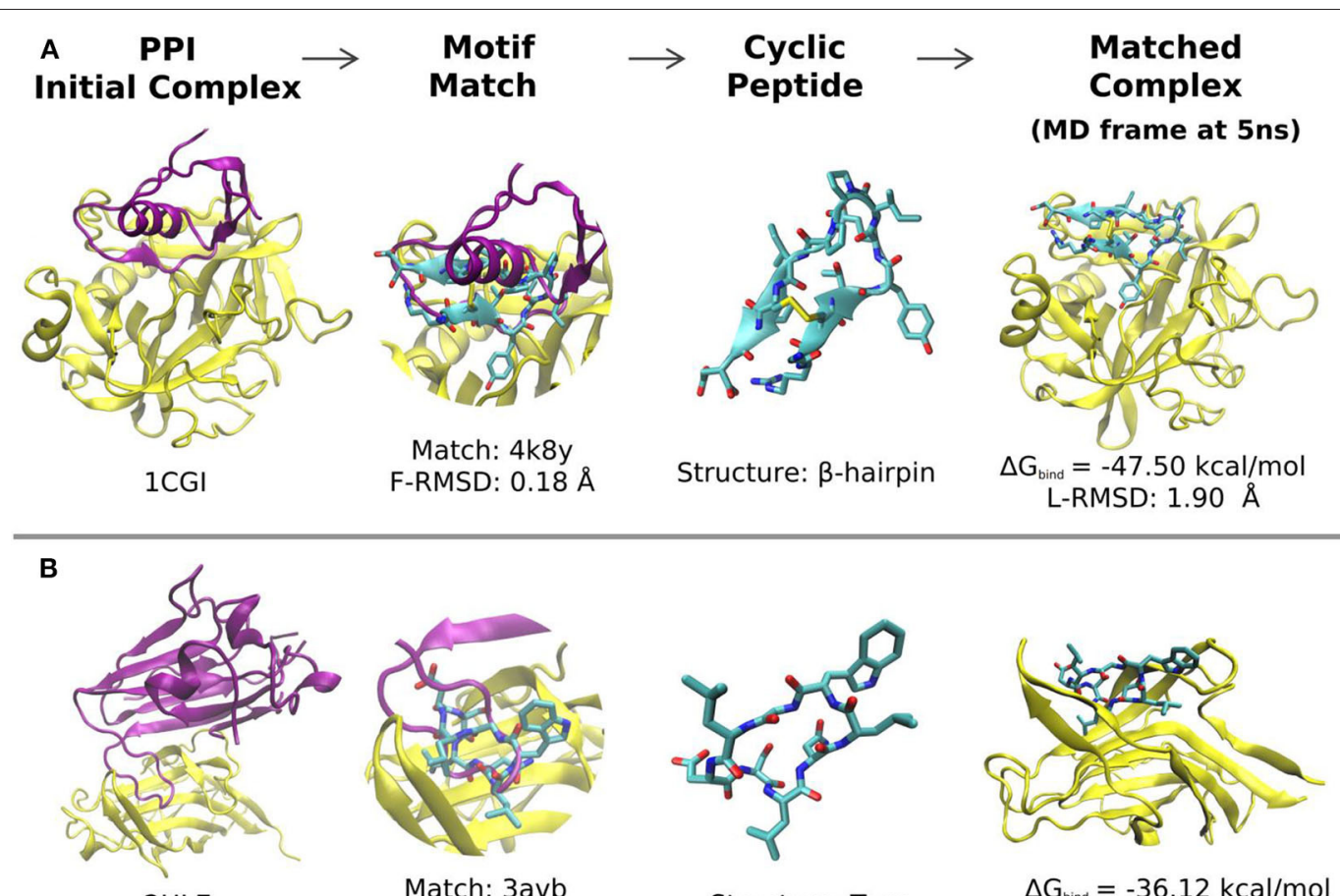

2HLE

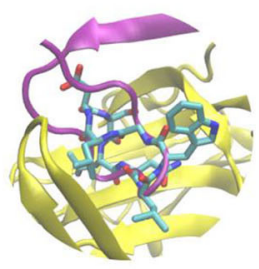

Match: $3 a v b$

F-RMSD: $0.31 \AA$

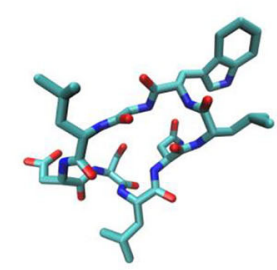

Structure: Turn

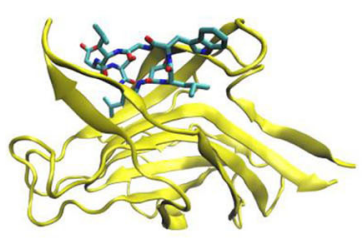
$\Delta \mathrm{G}_{\text {bind }}=-36.12 \mathrm{kcal} / \mathrm{mol}$
L-RMSD: $1.55 \AA$

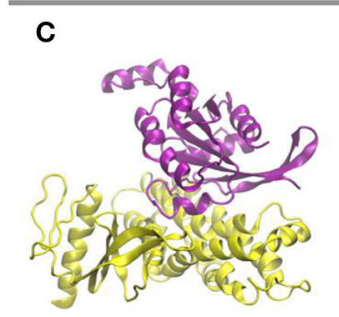

$2 N Z 8$

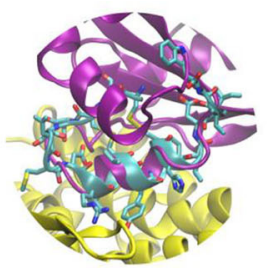

Match: $5 \mathrm{glh}$

F-RMSD: $0.18 \AA$

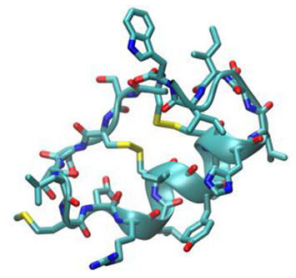

Structure: $\alpha$-helix

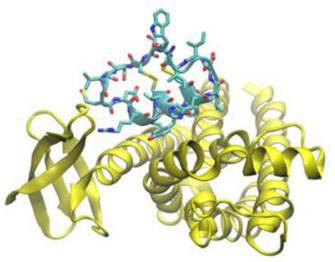

$\Delta \mathrm{G}_{\text {bind }}=-36.05 \mathrm{kcal} / \mathrm{mol}$

L-RMSD: $2.79 \AA$

FIGURE 3 | Representative matches and modeled structures of protein-cyclic-peptide complexes. (A) Example of a cyclic peptide with an $\beta$-hairpin motif at the interface (pdb-entries of the complex and the cyclic peptide template are indicated; the calculated MMGBSA interaction energy and final deviation from the start structure are also included). (B) Same as in (A) but for a cyclic peptide with a turn motif as binder. (C) Same as in (A,B) for a cyclic $\alpha$-helix binding motif. In each case the target protein-protein complex is shown as cartoon (yellow: receptor; pink: ligand protein). The superimposed matching cyclic peptide is indicated in the second column and the cyclic peptide (with adapted interface sequence) is shown in the third column. The last column represents the final structures of the receptor protein (yellow) in complex with the cyclic peptide after 5 ns MD simulation in explicit solvent.

simulation (Figures 4A-D). Overall, the calculated MMGBSA binding energies are close to the average for each case with a standard error of $<0.8 \mathrm{kcal} \mathrm{mol}^{-1}$ even when considering both independent simulations for each case supporting the robustness of the evaluation protocol.

In addition, we evaluated the importance of the generated interface side chains on the ligand-fitted cyclic peptides constructed by our cPEPmatch approach that are identical to the side chains of the native complex. Our evaluation procedure was applied to protein bound cyclic peptides containing the original sequence of the corresponding cyclic peptide from the data base or containing just alanine substitutions at the interface (Figure 5). The same set of representative cases (1cgi, 2cfh, 2hle, and $4 \mathrm{dg} 4)$ were used. The cyclic peptides with alanines at the interface proved to be considerably weaker binders in our MMGBSA evaluation (Figure 5) compared to the cyclic peptides with native interface residues when the secondary structure is an $\alpha$-helix or a turn (2hle, $2 \mathrm{nz} 8$, and $4 \mathrm{dg} 4$ ). However, for the case with the cyclic peptide providing a $\beta$-hairpin interface (1cgi) predicted binding was almost as strong as for the native sequence (Figure 5). In this case, however, a significant amount of backbone-backbone interactions that take place at the $\beta$-hairpin interfaces was observed which may not be perturbed by the alanine substitutions. Whereas, in three out of the four test cases the alanine substitution reduced the MMGBSA score using the original sequence of the cyclic peptides (from the data base) 
TABLE 2 | Results for the 30 top matches.

\begin{tabular}{|c|c|c|c|c|c|}
\hline \multirow{2}{*}{$\begin{array}{l}\text { PPI } \\
1 \text { E96 }\end{array}$} & \multirow{2}{*}{$\begin{array}{l}\text { Cyclic peptide } \\
\text { 2Iwu }\end{array}$} & \multirow{2}{*}{$\begin{array}{c}\text { F-RMSD } \\
\text { (Å) } \\
1.56\end{array}$} & \multicolumn{2}{|c|}{$\begin{array}{c}\text { MM/GBSA } \\
\Delta \text { Gbind (kcal/mol) }\end{array}$} & \multirow{2}{*}{$\begin{array}{c}\text { L-RMSD } \\
\qquad \begin{array}{c}\text { (Å) } \\
3.53\end{array}\end{array}$} \\
\hline & & & -66.46 & \pm 0.52 & \\
\hline $1 \mathrm{ACB}$ & $4 \mathrm{k} 8 \mathrm{y}$ & 0.11 & -50.78 & \pm 0.33 & 1.17 \\
\hline $1 \mathrm{AHW}$ & $1 \mathrm{qx} 9$ & 2.38 & -29.30 & \pm 0.54 & 3.42 \\
\hline 1AZS & $5 \mathrm{glh}$ & 0.17 & -46.10 & \pm 0.33 & 3.98 \\
\hline $1 \mathrm{BKD}$ & $5 \mathrm{glh}$ & 0.07 & -82.38 & \pm 0.46 & 2.43 \\
\hline 1CGI & $4 \mathrm{k} 8 \mathrm{y}$ & 0.18 & -47.50 & \pm 0.34 & 1.90 \\
\hline 1D6R & $4 \mathrm{k} 8 \mathrm{y}$ & 0.09 & -53.57 & \pm 0.60 & 2.07 \\
\hline 1E6E & $5 \mathrm{glh}$ & 0.12 & -42.75 & \pm 0.28 & 3.95 \\
\hline 1EAW & $4 k 8 y$ & 0.43 & -49.78 & \pm 0.35 & 2.23 \\
\hline 1FAK & 6pio & 1.44 & -36.27 & \pm 0.59 & 2.73 \\
\hline 1FSK & $3 p 8 f$ & 0.36 & -40.38 & \pm 0.29 & 2.36 \\
\hline $1 \mathrm{~K} 74$ & $5 \mathrm{glh}$ & 0.34 & -39.63 & \pm 0.33 & 4.77 \\
\hline 1LFD & 1ebp & 0.43 & -30.43 & \pm 0.33 & 4.00 \\
\hline 1NW9 & $5 \mathrm{glh}$ & 0.25 & -31.99 & \pm 0.43 & 4.51 \\
\hline 1PXV & $4 k 8 y$ & 0.18 & -40.25 & \pm 0.30 & 3.99 \\
\hline 1ROR & $4 \mathrm{k} 8 \mathrm{y}$ & 0.10 & -40.74 & \pm 0.38 & 1.89 \\
\hline 1VFB & $5 \mathrm{glh}$ & 0.18 & -34.36 & \pm 0.24 & 3.23 \\
\hline 2B4J & 1 npo & 0.13 & -29.26 & \pm 0.25 & 4.16 \\
\hline 2COL & $5 \mathrm{glh}$ & 0.05 & -54.09 & \pm 0.51 & 1.77 \\
\hline $2 \mathrm{CFH}$ & $5 \mathrm{glh}$ & 0.18 & -50.49 & \pm 0.41 & 2.53 \\
\hline 2FD6 & $2 \mathrm{lwt}$ & 0.75 & -37.97 & \pm 0.47 & 1.83 \\
\hline 2HLE & 3avb & 0.16 & -36.12 & \pm 0.25 & 1.55 \\
\hline 2NZ8 & $5 \mathrm{glh}$ & 0.18 & -36.05 & \pm 0.33 & 2.79 \\
\hline 2SNI & $3 p 8 f$ & 0.44 & -57.30 & \pm 0.26 & 1.11 \\
\hline 2W9E & $5 \mathrm{glh}$ & 0.11 & -40.54 & \pm 0.34 & 4.85 \\
\hline 3AAA & $5 \mathrm{glh}$ & 0.09 & -43.83 & \pm 0.39 & 1.35 \\
\hline 3BP8 & $3 p 8 f$ & 0.47 & -36.02 & \pm 0.23 & 3.41 \\
\hline 3EOA & $4 \mathrm{k} 8 \mathrm{y}$ & 0.23 & -41.55 & \pm 0.28 & 1.27 \\
\hline 3L5W & $5 \mathrm{glh}$ & 0.09 & -30.03 & \pm 0.27 & 1.02 \\
\hline 3V6Z & $5 \mathrm{glh}$ & 0,17 & -36.90 & \pm 0.52 & 3.38 \\
\hline
\end{tabular}

gave in all cases a strong reduction of the calculated MMGBSA binding energy (Figure 5, green bars, labeled wild type).

\section{CONCLUSIONS}

One possibility to create cyclic peptide variants based on known protein-protein interface structures is to identify a linear interface motif with an appropriate distance of terminal side chains or backbone atoms such that cyclization is possible. Ideally, such motifs give stable cyclic peptide binders. However, it can be difficult to predict the backbone structure of a cyclized peptide if one introduces a chemical backbone or side chain crosslink. The crosslink can destabilize a desired geometry such that the cyclic peptide does not resemble the structure of the interface motif. An advantage of our strategy is that we base the construction of a desired cyclic peptide on known stable (high resolution) cyclic template structures. Hence, the approach avoids the uncertainty on how well a select cyclization of a given motif resembles a desired backbone structure. It is applicable
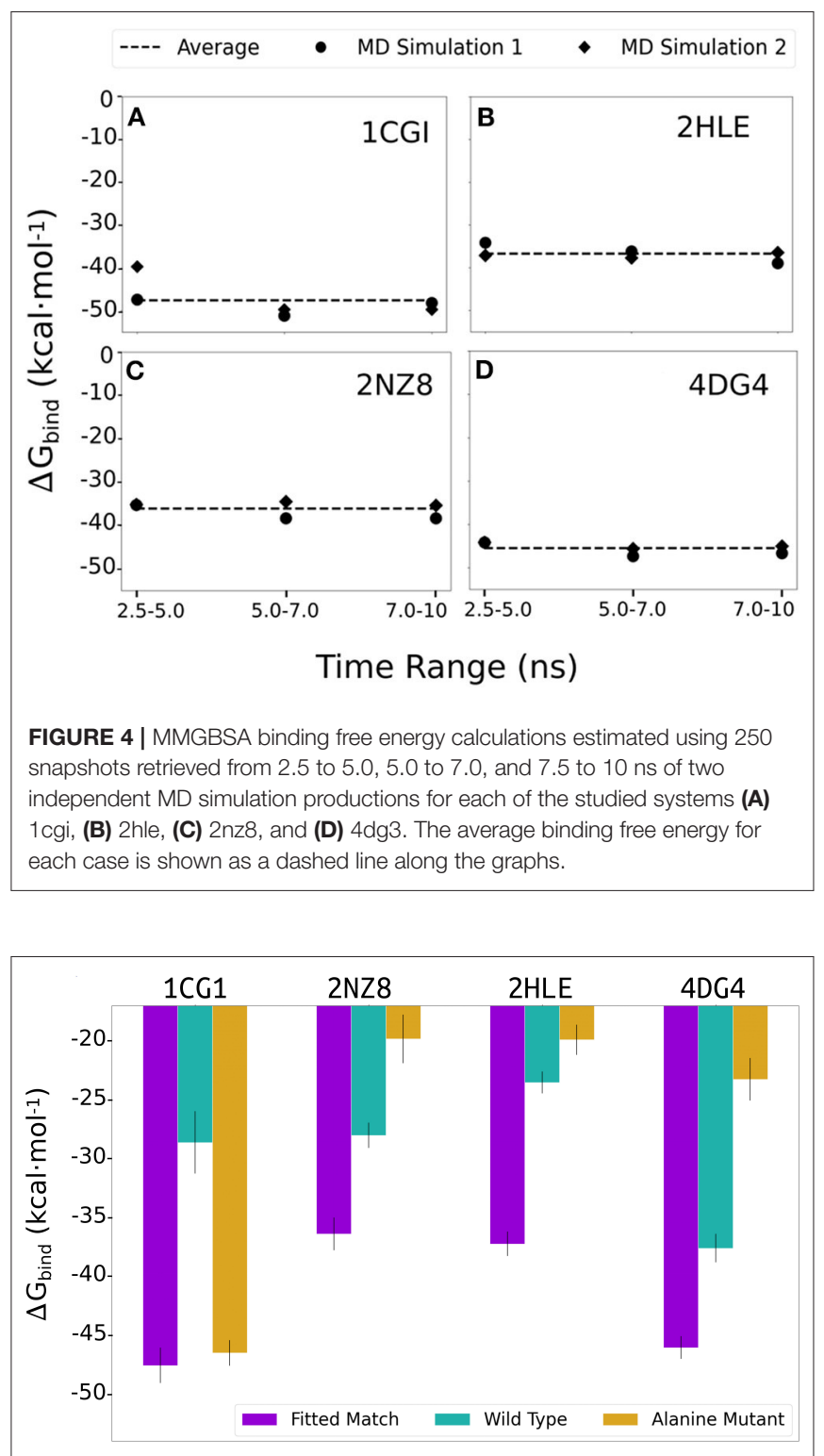

FIGURE 5 | Average of 3 MMGBSA binding energy calculations estimated using 250 snapshots retrieved from three intervals (2.5-5.0, 5.0-7.0, and $7.5-10 \mathrm{~ns}$ ) of MD simulations for the 1cgi, 2nz8, 2hle, and 4dg4 PPI receptors in complex with the cPEPmatch cyclic peptides and interface residues copied from the native complex (purple bar), or using the original cyclic peptide sequence (wild type) from the data base (cyan bar) or alanine substitutions for all interface residues of the cyclic peptide (orange bar).

if the structure of a protein-protein complex is known and even potentially useful if the receptor is flexible and adopts a different structure in the unbound form (as long as it can adopt the structure in the bound form). Most related to our method is an approach by Duffy et al. (2015) to identify both protein-protein interactions suitable for inhibition by cyclic peptides and the accompanying cyclic peptides that represent promising lead compounds. This approach is, however, based on a pharmacophore matching of the PPI interface region with 
corresponding side chains on sets of cyclic peptides whereas our method is entirely based on structural matching of the interface backbone onto segments of cyclic peptides with known structure. It is complementary also to other approaches to identify cyclic peptides that target PPI (Schreiber and Crabtree, 1992; Dechantsreiter et al., 1999; Sulyok et al., 2001; Shi et al., 2004; Zhang et al., 2014; Siegert et al., 2016; Shin et al., 2017).

The initial screen of our approach (without explicit solvent refinement) is extremely rapid, hundreds of PPI can be screened within a few seconds for cyclic peptides that match to backbone structures at the PPI interface. Interestingly, with a relatively small set of cyclic peptide templates it is possible to identify cyclic peptide-protein complexes that are stable during explicit solvent MD simulations and indicate very favorable interaction energy scores comparable or better than for known stable peptide-protein complexes. We also found that substitution by alanine in the evaluation could be a useful strategy in order to check the contribution of the side chains copied from the native interface to the cyclic peptide-protein interface to the stability of the complexes. It should be noted that a short MD simulation as used in our approach is useful to relax the complex structure but not long enough to exhaustively sample the complex conformations. The putative cyclic peptide modulators could be further improved by individual postmodifications and by studying specific cases experimentally. It could also be combined with approaches to identify peptide epitopes specific for mediating protein-protein interactions as well as allosteric sites our unstable loop segments in protein interaction regions (Paladino et al., 2020; Serapian and Colombo, 2020). Disruption of interactions that involve such regions often interferes with specific functions of the corresponding proteinprotein complex. Our cPEPmatch method, although at an early

\section{REFERENCES}

Arkin, M. R., Tang, Y., and Wells, J. A. (2014). Small-molecule inhibitors of protein-protein interactions: progressing toward the reality. Chem. Biol. 21, 1102-1114. doi: 10.1016/j.chembiol.2014.09.001

Bahadur, R. P., and Zacharias, M. (2008). The interface of protein-protein complexes: analysis of contacts and prediction of interactions. Cell. Mol. Life Sci. 65, 1059-1072. doi: 10.1007/s00018-007-7451-x

Case, D. A., Cheatham, T. E., Darden, T., Gohlke, H., Luo, R., Merz, K. M., et al. (2005). The Amber biomolecular simulation programs. J. Comput. Chem. 26, 1668-1688. doi: 10.1002/jcc. 20290

Che, Y. (2019). "Design of cyclic peptides as protein recognition motifs," in Cyclic Peptide Design, ed G. Goetz (New York, NY: Springer), 97-106. doi: 10.1007/978-1-4939-9504-2_6

Chen, F., Liu, H., Sun, H., Pan, P., Li, Y., Li, D., et al. (2016). Assessing the performance of the MM/PBSA and MM/GBSA methods. 6. Capability to predict protein-protein binding free energies and re-rank binding poses generated by protein-protein docking. Phys. Chem. Chem. Phys. 18, 22129-22139. doi: 10.1039/C6CP03670H

Conte, L. L., Chothia, C., and Janin, J. (1999). The atomic structure of protein-protein recognition sites. J. Mol. Biol. 285, 2177-2198. doi: 10.1006/jmbi.1998.2439

Dechantsreiter, M. A., Planker, E., Math,ä, B., Lohof, E., Hölzemann, G., Jonczyk, A., et al. (1999). N-methylated cyclic RGD peptides as highly active and selective $\alpha(\mathrm{v}) \beta 3$ integrin antagonists. J. Med. Chem. 42, 3033-3040. doi: $10.1021 /$ jm970832g stage, has demonstrated the ability to be an easy automatic step toward identifying putative cyclic peptide templates for PPI modulators that could form the basis for subsequent experimental testing or more extensive analysis of the interface including the hydration properties. In the future, a larger and even more diverse set cyclic peptides will be constructed and some of the putative cyclic peptides will be tested experimentally.

\section{AUTHOR CONTRIBUTIONS}

MZ designed and supervised research. BS performed research and analyzed data. All authors contributed to writing of the manuscript.

\section{FUNDING}

This research was conducted within the Max Planck School Matter to Life supported by the German Federal Ministry of Education and Research (BMBF) in collaboration with the Max Planck Society. We acknowledge also support by the Leibniz super computer (LRZ) center for providing supercomputer support by grant pr27za.

\section{ACKNOWLEDGMENTS}

We thank Shu-Yu Chen and P. Marlow for helpful discussion.

\section{SUPPLEMENTARY MATERIAL}

The Supplementary Material for this article can be found online at: https://www.frontiersin.org/articles/10.3389/fchem. 2020.573259/full\#supplementary-material

Driggers, E. M., Hale, S. P., Lee, J., and Terrett, N. K. (2008). The exploration of macrocycles for drug discovery - an underexploited structural class. Nat. Rev. Drug Discov. 7:2590. doi: 10.1038/nrd2590

Duffy, F., Maheshwari, N., Buchete, N.-V., and Shields, D. (2019). “Computational opportunities and challenges in finding cyclic peptide modulators of proteinprotein interactions," in Cyclic Peptide Design, ed G. Goetz (New York, NY: Springer), 73-95. doi: 10.1007/978-1-4939-9504-2_5

Duffy, F. J., O’Donovan, D., Devocelle, M., Moran, N., OŠConnell, D. J., and Shields, D. C. (2015). Virtual screening using combinatorial cyclic peptide libraries reveals protein interfaces readily targetable by cyclic peptides. J. Chem. Inf. Model. 55, 600-613. doi: 10.1021/ci500431q

Fontaine, F., Overman, J., and François, M. (2015). Pharmacological manipulation of transcription factor protein-protein interactions: opportunities and obstacles. Cell Regen 4:2. doi: 10.1186/s13619-015-0015-x

Fry, D. C. (2006). Protein-protein interactions as targets for small molecule drug discovery. Biopolymers 84, 535-552. doi: 10.1002/bip.20608

Gavenonis, J., Sheneman, B. A., Siegert, T. R., Eshelman, M. R., and Kritzer, J. A. (2014). Comprehensive analysis of loops at protein-protein interfaces for macrocycle design. Nat. Chem. Biol. 10, 716-722. doi: 10.1038/nchembio. 1580

Grauer, A., and König, B. (2009). Peptidomimetics - a versatile route to biologically active compounds. Eur. J. Org. Chem. 5099-5111. doi: 10.1002/ejoc.2009 00599

Huigens, R. W., Morrison, K. C., Hicklin, R. W., Timothy, T. A., Richter, M. F., and Hergenrother, P. J. (2013). A ring-distortion strategy to construct stereochemically complex and structurally diverse compounds from natural products. Nat. Chem. 5, 195-202. doi: 10.1038/nchem.1549 
Jorgensen, W. L., Chandrasekhar, J., Madura, J. D., Impey, R. W., and Klein, M. L. (1983). Comparison of simple potential functions for simulating liquid water. J. Chem. Phys. 79, 926-935. doi: 10.1063/1.445869

Kallen, J., Mikol, V., Taylor, P., and D.walkinshaw, M. (1998). X-ray structures and analysis of 11 cyclosporin derivatives complexed with cyclophilin A. J. Mol. Biol. 283, 435-449. doi: 10.1006/jmbi.1998.2108

Keskin, O., Ma, B., and Nussinov, R. (2005). Hot regions in protein-protein interactions: the organization and contribution of structurally conserved hot spot residues. J. Mol. Biol. 345, 1281-1294. doi: 10.1016/j.jmb.2004.10.077

Kuenemann, M. A., Sperandio, O., Labbé, C. M., Lagorce, D., Miteva, M. A., and Villoutreix, B. O. (2015). In silico design of low molecular weight proteinprotein interaction inhibitors: Overall concept and recent advances. Prog. Biophys. Mol. Biol. 119, 20-32. doi: 10.1016/j.pbiomolbio.2015.02.006

London, N., Raveh, B., Movshovitz-Attias, D., and Schueler-Furman, O. (2010). Can self-inhibitory peptides be derived from the interfaces of globular protein-protein interactions? Proteins Struct. Funct. Bioinforma. 78, 3140-3149. doi: $10.1002 /$ prot. 22785

Maier, J. A., Martinez, C., Kasavajhala, K., Wickstrom, L., Hauser, K. E., and Simmerling, C. (2015). ff14SB: Improving the accuracy of protein side chain and backbone parameters from ff99SB. J. Chem. Theory Comput. 11, 3696-3713. doi: 10.1021/acs.jctc.5b00255

Mallinson, J., and Collins, I. (2012). Macrocycles in New Drug Discovery. London: Future Science Ltd London. doi: 10.4155/fmc. 12.93

Marsault, E., and Peterson, M. L. (2011). Macrocycles are great cycles: Applications, opportunities, and challenges of synthetic macrocycles in drug discovery. J. Med. Chem. 54, 1961-2004. doi: 10.1021/jm1012374

Martins, M. B., and Carvalho, I. (2007). Diketopiperazines: biological activity and synthesis. Tetrahedron 63, 9923-9932. doi: 10.1016/j.tet.2007.04.105

Metz, A., Pfleger, C., Kopitz, H., Pfeiffer-Marek, S., Baringhaus, K. H., and Gohlke, H. (2012). Hot spots and transient pockets: predicting the determinants of small-molecule binding to a protein-protein interface. J. Chem. Inf. Model. 52, 120-133. doi: 10.1021/ci200322s

Milhas, S., Raux, B., Betzi, S., Derviaux, C., Roche, P., Restouin, A., et al. (2016). Protein-protein interaction inhibition (2P2I)-oriented chemical library accelerates hit discovery. ACS Chem. Biol. 11, 2140-2148. doi: $10.1021 /$ acschembio.6b00286

Nevola, L., and Giralt, E. (2015). Modulating protein-protein interactions: the potential of peptides. Chem. Commun. 51, 3302-3315. doi: 10.1039/C4CC08565E

Paladino, A., Woodford, M. R., Backe, S. J., Sager, R. A., Kancherla, P., Daneshvar, M. A., et al. (2020). Chemical perturbation of oncogenic protein folding: from the prediction of locally unstable structures to the design of disruptors of Hsp90-client interactions. Chem. - Eur. J. 26, 9459-9465. doi: $10.1002 /$ chem.202000615

Qian, Z., Dougherty, P. G., and Pei, D. (2017). Targeting intracellular proteinprotein interactions with cell-permeable cyclic peptides. Curr. Opin. Chem. Biol. 38, 80-86. doi: 10.1016/j.cbpa.2017.03.011

Rezai, T., Yu, B., Millhauser, G. L., Jacobson, M. P., and Lokey, R. S. (2006). Testing the conformational hypothesis of passive membrane permeability using synthetic cyclic peptide diastereomers. J. Am. Chem. Soc. 128, 2510-2511. doi: $10.1021 / \mathrm{ja} 0563455$

Ryan, D. P., and Matthews, J. M. (2005). Protein-protein interactions in human disease. Curr. Opin. Struct. Biol. 15, 441-446. doi: 10.1016/j.sbi.2005.06.001
Ryckaert, J.-P., Ciccotti, G., and Berendsen, H. J. C. (1977). Numerical integration of the cartesian equations of motion of a system with constraints: molecular dynamics of n-alkanes. J. Comput. Phys. 23, 327-341. doi: 10.1016/0021-9991(77)90098-5

Schreiber, S. L., and Crabtree, G. R. (1992). The mechanism of action of cyclosporin A and FK506. Immunol. Today 13, 136-142. doi: 10.1016/0167-5699(92)90111-J

Serapian, S. A., and Colombo, G. (2020). Designing Molecular Spanners to Throw in the Protein Networks. Chem. Eur. J. 26, 4656-4670. doi: 10.1002/chem.201904523

Shi, Z. D., Wei, C. Q., Lee, K., Liu, H., Zhang, M., Araki, T., et al. (2004), Macrocyclization in the design of non-phosphorus-containing Grb2 SH2 domain-binding ligands. J. Med. Chem. 47, 2166-2169. doi: 10.1021/jm030510e

Shin, W.-H., Christoffer, C. W., and Kihara, D. (2017). In silico structure-based approaches to discover protein-protein interaction-targeting drugs. Methods 131, 22-32. doi: 10.1016/j.ymeth.2017.08.006

Siegert, T. R., Bird, M. J., Makwana, K. M., and Kritzer, J. A. (2016). Analysis of loops that mediate protein-protein interactions and translation into submicromolar inhibitors. J. Am. Chem. Soc. 138, 12876-12884. doi: 10.1021/jacs.6b05656

Sulyok, G. A. G., Gibson, C., Goodman, S. L., Hölzemann, G., Wiesner, M., and Kessler, H. (2001). Solid-phase synthesis of a nonpeptide rgd mimetic library: new selective $\alpha \mathrm{v} \beta 3$ integrin antagonists. J. Med. Chem. 44, 1938-1950. doi: 10.1021/jm0004953

Tikk, D., Thomas, P., Palaga, P., Hakenberg, J., and Leser, U. (2010). A Comprehensive benchmark of kernel methods to extract proteinprotein interactions from literature. PLoS Comput. Biol. 6:1000837. doi: 10.1371/journal.pcbi.1000837

Villoutreix, B. O., Kuenemann, M. A., Poyet, J. L., Bruzzoni-Giovanelli, H., Labbé, C., Lagorce, D., et al. (2014). Drug-like protein-protein interaction modulators: challenges and opportunities for drug discovery and chemical biology Wiley- $\mathrm{VCH}$ Verlag. doi: 10.1002/minf.201400040

Wang, C., Greene, D., Xiao, L., Qi, R., and Luo, R. (2018). Recent developments and applications of the MMPBSA method. Front. Mol. Biosci. 4:87. doi: $10.3389 /$ fmolb.2017.00087

Wells, J. A., and McClendon, C. L. (2007). Reaching for high-hanging fruit in drug discovery at protein-protein interfaces. Nature 450, 1001-1009. doi: 10.1038 /nature06526

Zhang, Z., Lin, Z., Zhou, Z., Shen, H. C., Yan, S. F., Mayweg, A. V., et al. (2014). Structure-based design and synthesis of potent cyclic peptides inhibiting the YAP-TEAD protein-protein interaction. ACS Med. Chem. Lett. 5, 993-998. doi: $10.1021 / \mathrm{ml} 500160 \mathrm{~m}$

Conflict of Interest: The authors declare that the research was conducted in the absence of any commercial or financial relationships that could be construed as a potential conflict of interest.

Copyright $\odot 2020$ Santini and Zacharias. This is an open-access article distributed under the terms of the Creative Commons Attribution License (CC BY). The use, distribution or reproduction in other forums is permitted, provided the original author(s) and the copyright owner(s) are credited and that the original publication in this journal is cited, in accordance with accepted academic practice. No use, distribution or reproduction is permitted which does not comply with these terms. 